\title{
Looseness of plane graphs
}

\author{
Július Czap, Stanislav Jendrol, \\ František Kardoš, and Jozef Miškuf \\ Institute of Mathematics \\ P. J. Šafárik University \\ Jesenná 5, SK-04001 Košice, Slovakia
}

e-mail: julius.czap@upjs.sk, stanislav.jendrol@upjs.sk,
frantisek.kardos@upjs.sk, jozef.miskuf@upjs.sk

\begin{abstract}
A face of a vertex coloured plane graph is called loose if the number of colours used on its vertices is at least three. The looseness of a plane graph $G$ is the minimum $k$ such that any surjective $k$-colouring involves a loose face. In this paper we prove that the looseness of a connected plane graph $G$ equals the maximum number of vertex disjoint cycles in the dual graph $G^{*}$ increased by 2 .

We also show upper bounds on the looseness of graphs based on the number of vertices, the edge connectivity, and the girth of the dual graphs. These bounds improve the result of Negami for the looseness of plane triangulations. We also present infinite classes of graphs where the equalities are attained.
\end{abstract}

Keywords: vertex colouring, loose colouring, looseness, plane graph, dual graph

2010 Mathematics Subject Classification: 05C10, 05C15, 05C38

\section{Introduction}

We use the standard terminology according to Bondy and Murty [2], except for few notations defined throughout. However, we recall some frequently used terms. All considered graphs are finite, loops and multiple edges are allowed.

Let $G=(V, E, F)$ be a connected plane graph with the vertex set $V$, the edge set $E$ and the face set $F$. The degree of a vertex $v$ is the number of edges incident with $v$, each loop counting as two edges. For a face $f$, the size of $f$, $\operatorname{deg}(f)$, is defined to be the length of the shortest closed walk containing all edges from the boundary of $f$. 
We write $v \in f$ if a vertex $v$ is incident with a face $f$. The vertices $u$ and $v$ are face independent if there is no face $f$ such that $v \in f$ and $u \in f$. A set $S$ of vertices is face independent if any two vertices from $S$ are face independent. The face independence number of a graph $G, \alpha_{2}(G)$, is defined to be the number of vertices in the maximum face independent set of vertices.

A $k$-colouring of a graph $G$ is a mapping $\varphi: V(G) \rightarrow\{1, \ldots, k\}$. An edge in a vertex coloured graph is heterochromatic if its ends are coloured with different colours. Otherwise it is monochromatic.

Colourings of graphs embedded on surfaces with face-constrains have recently drawn a substantial amount of attention. There are three questions derived from hypergraph colourings that one may ask in this setting:

Question 1 What is the minimum number of colours $r b(G)$ that any surjective vertex colouring of a connected plane graph $G$ with $r b(G)$ colours enforces a face with vertices of mutually distinct colours, i.e., there is a rainbow face?

Question 2 What is the minimum number of colours needed to colour a connected plane graph in such a way that each of its faces is incident with vertices of at least two different colours, i.e., there is no monochromatic face?

Question 3 What is the maximum number of colours $k(G)$ that can be used in a colouring of a connected plane graph $G$ in such a way that every face is monochromatic or bichromatic, i.e., there is no loose face?

Now we survey some results dealing with Question 1. The parameter $\operatorname{rb}(G)$ is called the rainbowness of $G$, see [6]. Ramamurthi and West $[13,14]$ noticed that every plane graph $G$ of order $n$ has a nonrainbow colouring with at least $\left\lceil\frac{n}{4}\right\rceil+1$ colours by the Four Colour Theorem. They had conjectured [14] and Jungič et al. [7] proved that this bound can be improved to the bound $\left\lceil\frac{n}{2}\right\rceil+1$ for triangle-free plane graphs. More generally, Jungič et al. [7] proved that every plane graph of order $n$ with girth $g \geq 5$ has a nonrainbow colouring with at least $\left\lceil\frac{g-3}{g-2} n-\frac{g-7}{2(g-2)}\right\rceil$ colours if $g$ is odd and $\left\lceil\frac{g-3}{g-2} n-\frac{g-6}{2(g-2)}\right\rceil$ if $g$ is even. It is also shown that these bounds are best possible.

There are also results concerning upper bounds on $r b(G)$. Dvořák et al. [4] proved that for every $n$ vertex 3 -connected plane graph $G$ it holds $\operatorname{rb}(G) \leq$ $\left\lfloor\frac{7 n+1}{9}\right\rfloor$, for every 4-connected graph $G$ it holds $r b(G) \leq\left\lfloor\frac{5 n+2}{8}\right\rfloor$ if $n \not \equiv 3(\bmod 8)$, $r b(G) \leq\left\lfloor\frac{5 n-6}{8}\right\rfloor$ if $n \equiv 3(\bmod 8)$ and for every 5 -connected plane graph $G$ $r b(G) \leq\left\lfloor\frac{43}{100} n+\frac{6}{25}\right\rfloor$. Moreover, the bounds for the 3- and 4-connected graphs are best possible.

The second of these problems is a classical hypergraph colouring problem. The edges of the face hypergraph of a plane graph are the vertex sets of the faces. The Four Colour Theorem [1] implies that the face hypergraph of a simple plane graph has chromatic number 2 , as follows. Let $G$ be a simple plane graph. Add 
edges to $G$ to obtain a triangulation $T$. $T$ has a proper colouring $\psi$ which uses at most 4 colours. Combine the first colour class with the second, and combine the third with the fourth. This yields a 2-colouring $\varphi$ of a graph $G$. It is easy to check that $\varphi$ is a required 2-colouring of $G$.

It is worth noting that there are other proofs of this bound that do not require the Four Colour Theorem. We present Penaud's [12] elegant proof:

We can assume that the given graph $G$ is a triangulation. The dual graph $G^{*}$ is a 2-connected, 3-regular graph, since $G$ is a loopless triangulation. By Petersen's Theorem (see [2], pp. 431), $G^{*}$ has a 2 -factor $H^{*}$, i.e., a decomposition of its vertex set into cycles. Let $\varphi(v)=1$ if $v$ is contained in the interior of an even number of cycles of $H^{*}, \varphi(v)=2$ otherwise.

We claim that for such colouring $\varphi$ there is no monochromatic face in $G$. Consider a face uvw in $G$. There is a pair of dual edges crossing this face, which belong to the 2 -factor we obtained. These edges separate one of the vertices, say $u$, from the other two vertices in the face. Hence, the parity of the number of cycles containing $u$ is different from the parity of the number of cycles containing $v$ and $w$.

Let us mention that colourings that avoid both monochromatic and rainbow faces have been also studied, see e.g. [3, 8, 9].

In this paper, we focus on Question 3. Let $\varphi$ be a $k$-colouring of a plane graph $G=(V, E, F)$. For a set $X \subseteq V$ we define $\varphi(X)$ to be the set of colours $\{\varphi(v) ; v \in X\}$. Particularly, if $f$ is a face of $G$ then $\varphi(f)$ denotes the set of colours used on the vertices incident with the face $f$. A face $f \in F$ is called loose if $|\varphi(f)| \geq 3$, otherwise it is called nonloose. A $k$-colouring of a graph $G$ is called the loose $k$-colouring if it involves any loose face, otherwise it is a nonloose $k$-colouring.

Negami addressed the following anti-Ramsey extremal question (equivalent to our third one):

What is the smallest number $l s(G)$ of colours such that every vertex colouring of a plane graph $G$ with $l s(G)$ colours enforces a face incident with three vertices coloured by three different colours, i.e., $G$ contains a loose face ?

The invariant $l s(G)$ of a plane graph $G$ is called the looseness of $G$. The notion of looseness has been introduced by Negami and Midorikawa [11], see also [10]. They have shown lower and upper bounds for the looseness of a triangulation in terms of the independence number $\alpha_{0}(G)$, as follows: $\alpha_{0}(G)+2 \leq l s(G) \leq$ $2 \alpha_{0}(G)+1$.

It is easy to see that $l s(G)=k(G)+1$ and the results obtained in either of the scenarios translate smoothly to the other one. Hence, in this article we use the terminology introduced by Negami.

The looseness is well defined for all plane graphs having at least one face incident with at least three different vertices. Throughout the paper, we will 
consider only such graphs.

The rest of the paper is organized as follows. In Section 2 we prove that the problem of determining the looseness of connected plane graph $G$ is equivalent to the problem of determining the maximum number of vertex disjoint cycles in the dual graph $G^{*}$. The latter problem is known to be NP-hard, see [5], so good estimations for this parameter are welcome.

Section 3 deals with upper bounds on the looseness of plane graphs in terms of basic graph invariants, namely the girth and the edge connectivity. We show that if the girth of the dual graph $G^{*}$ is $g$ then the looseness is at most $\frac{1}{g}|F(G)|+2$, moreover, this bound is tight.

We use the fact that the edge connectivity $\kappa^{\prime}$ of a plane graph $G$ has a close relation to the girth of the dual graph $G^{*}$ to prove that $l s(G) \leq \frac{1}{\kappa^{\prime}}|F(G)|+2$, moreover, the bound is best possible. We also show that if $G$ is a 3-connected cubic plane $n$-vertex graph then $l s(G) \leq \frac{1}{6} n+\frac{8}{3}$.

In Section 4 we prove that if $G$ is a connected simple plane graph on $n$ vertices, then the looseness of $G$ is at most $\frac{2 n+2}{3}$. This bound is best possible for classes of 1-, 2-, and 3-connected plane graphs.

Finally, observe that if a plane graph $G$ contains only triangular faces then $r b(G)=l s(G)=k(G)+1$. The upper bound for the looseness (and hence for the rainbowness) of plane triangulations known so far was $2 \alpha_{0}+1$, where $\alpha_{0}$ is the independence number. In the paper this bound is improved to $\frac{n+2}{2}$ if the minimum vertex degree is 4 and $\frac{2 n+6}{5}$ if the minimum vertex degree is 5 , where $n$ is the number of vertices. For triangulations with vertices of degree 3 our bound is $\frac{2 n+2}{3}$. There are triangulations where the bound $\frac{2 n+2}{3}$ is smaller than $2 \alpha_{0}+1$ and vice versa, but it is well known that the problem to compute the independence number of plane graphs is NP-hard. All presented upper bounds are tight. These results are summarized in Section 5.

\section{General properties of the loose colourings}

Consider a surjective $k$-colouring of a connected plane graph $G=(V, E, F)$.

Proposition 2.1 Let $G$ be a plane graph. Then $l s(G) \geq \alpha_{2}(G)+2$.

\section{Proof}

We obtain a suitable nonloose $\left(\alpha_{2}(G)+1\right)$-colouring of $G$ in the following way: Colour the face-independent vertices with colours $2, \ldots, \alpha_{2}(G)+1$, and the remaining vertices with colour 1 .

We proceed with the other estimations on looseness of plane graphs.

Let us recall that the (geometric) dual $G^{*}=\left(V^{*}, E^{*}, F^{*}\right)$ of the plane graph $G=(V, E, F)$ can be defined as follows (see [2], pp. 252): Corresponding to each 
face $f$ of $G$ there is a vertex $f^{*}$ of $G^{*}$, and corresponding to each edge $e$ of $G$ there is an edge $e^{*}$ of $G^{*}$; two vertices $f^{*}$ and $g^{*}$ are joined by the edge $e^{*}$ in $G^{*}$ if and only if their corresponding faces $f$ and $g$ are separated by the edge $e$ in $G$ (an edge separates the faces incident with it).

First we show a lower bound on the looseness.

Theorem 2.1 Let $G=(V, E, F)$ be a connected plane graph such that the dual $G^{*}$ of $G$ has $t$ vertex disjoint cycles. Then

$$
l s(G) \geq t+2 \text {. }
$$

\section{Proof}

Let $\mathcal{C}=\left\{C_{1}, \ldots, C_{t}\right\}$ be a set of vertex disjoint cycles in $G^{*}$. Each $C_{i}$ divides the plane into two parts, let $D_{i}$ be the bounded one.

Since the cycles in $\mathcal{C}$ are vertex disjoint, for each $1 \leq i<j \leq t$ we have either $D_{i} \cap D_{j}=\emptyset$, or $D_{i} \subseteq D_{j}$, or vice versa. We say that $C_{i}$ is inside $C_{j}$ (and write $C_{i} \preceq C_{j}$ ) if $D_{i} \subseteq D_{j}$, for $1 \leq i, j \leq t$. It is easy to see that $\preceq$ is reflexive, antisymmetric and transitive relation on $\mathcal{C}$, thus $(\mathcal{C}, \preceq)$ is a partially ordered set. We can extend the ordering into a linear ordering. Therefore, without loss of generality we can assume that the cycles in $\mathcal{C}$ are indexed in such a way that $C_{i} \preceq C_{j}$ implies $i \leq j$.

Given the cycles $\mathcal{C}$, we colour the faces of $G^{*}$ in the following way: Faces

contained in $D_{i} \backslash \bigcup_{s=1}^{i-1} D_{s}$ receive the colour $i$; faces not contained in any $D_{i}$ receive the colour $t+1$. It is clear that this colouring induces the colouring of the vertices of the graph $G$ using precisely $t+1$ colours. Observe that all the faces inside $C_{i}$ (the faces forming $D_{i}$ ) received the colours at most $i$.

We show that this colouring is a nonloose $(t+1)$-colouring. Let us suppose for a contradiction that the graph $G$ contains a face $f$ which is incident with three vertices $v_{i}, v_{j}, v_{k}$ having three different colours $1 \leq i<j<k \leq t+1$. These correspond to three faces $v_{i}^{*}, v_{j}^{*}, v_{k}^{*}$ of $G^{*}$ coloured $i, j, k$, all incident with a vertex $f^{*}$. Since the face $v_{i}^{*}$ has colour $i, v_{i}^{*} \subseteq D_{i}$. On the other hand, the face $v_{j}^{*}$ has colour $j>i$, therefore $v_{j}^{*}$ is not inside $C_{i}$. The vertex $f^{*}$ is incident both with a face inside and outside $C_{i}$, hence $f^{*} \in C_{i}$. Analogously, $v_{j}^{*}$ is inside $C_{j}, v_{k}^{*}$ is outside $C_{j}$, hence $f^{*} \in C_{j}$. The vertex $f^{*}$ lies on two vertex disjoint cycles, a contradiction.

Theorem 2.2 Let $G=(V, E, F)$ be a connected plane graph and let $G^{*}$ be its dual. Then there are $t_{0}$ vertex disjoint cycles in $G^{*}$ such that

$$
l s(G)=t_{0}+2 .
$$

\section{Proof}

Let $\varphi$ be a nonloose $k$-colouring of the graph $G$, such that $k=l s(G)-1$. Let $G^{*}=\left(V^{*}, E^{*}, F^{*}\right)$ be the dual of $G$. 
Let $E_{i j}$ denote the set of edges of the graph $G$ such that their ends are coloured with colours $i$ and $j, i \neq j$. Let $E_{i j}^{*}$ be the set of edges in the dual graph $G^{*}$ which correspond to $E_{i j}$ in $G$.

The graph $G^{*}\left[E_{i j}^{*}\right]$ induced by the edges from $E_{i j}^{*}$ has minimum degree at least two, because if a face $f \in F(G)$ is incident with a two coloured edge then its boundary walk contains at least two such edges. Hence, each graph $G^{*}\left[E_{i j}^{*}\right]$ contains at least one cycle, say $C_{i j}$. Moreover, each vertex $v^{*} \in V\left(G^{*}\right)$ can be incident with at most one type of heterochromatic edges, otherwise the face corresponding to $v^{*}$ would be incident with at least three vertices coloured differently. Hence,

$$
G^{*}\left[E_{i j}^{*}\right] \cap G^{*}\left[E_{l k}^{*}\right]=\emptyset \text { for }\{i, j\} \neq\{k, l\} .
$$

From (1) follows that the cycles $C_{i j}$ and $C_{k l}$ are vertex disjoint for $\{i, j\} \neq$ $\{k, l\}$. Let $t_{0}$ denote the number of such cycles. Applying Theorem 2.1 we have $t_{0} \leq l s(G)-2$.

The colouring $\varphi$ uses $k$ colours, therefore the graph $G$ has at least $k-1$ types of heterochromatic edges. Hence, $t_{0} \geq k-1=l s(G)-2$. So $t_{0}=l s(G)-2$.

\section{Looseness, girth and edge-connectivity}

Let us recall that the girth of a graph $G$ is the length of its shortest cycle.

Theorem 3.1 Let $G=(V, E, F)$ be a connected plane graph, let $g$ be the girth of the dual graph $G^{*}$ of $G$. Then

$$
l s(G) \leq \frac{1}{g}|F(G)|+2 .
$$

Moreover, the bound is sharp.

\section{Proof}

Theorem 2.2 implies that the dual graph $G^{*}$ of a graph $G$ contains $t$ vertex disjoint cycles such that $l s(G)=t+2$. Clearly, each cycle contains at least $g$ vertices. Hence, we get $t \leq \frac{|F(G)|}{g}$. So we are done.

To prove the sharpness of the bound, it suffices to find examples of plane graphs such that

$$
g \cdot \alpha_{2}(G) \geq|F(G)|
$$

Then, by Proposition 2.1 we have $l s(G) \geq \alpha_{2}(G)+2 \geq \frac{1}{g}|F(G)|+2$.

Let $g \geq 2$ be fixed. Let $G_{0}$ be a plane drawing of the complete bipartite graph $K_{2, k}\left(k \geq \frac{g}{2}\right)$ such that all faces are quadrangles. Let $u_{1}$ and $u_{2}$ be the two vertices of degree $k$ and $v_{1}, \ldots, v_{k}$ be the vertices of degree 2 . 


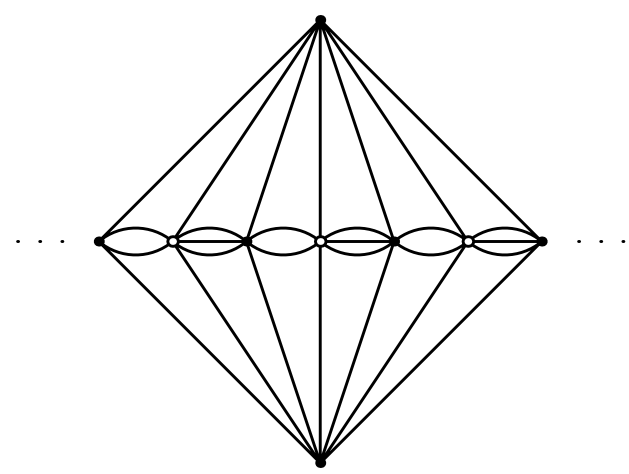

Figure 1: An example of a graph where inequality (2) is satisfied, for $g=7$.

For $i=1, \ldots, k$ we insert a vertex $w_{i}$ into the face bounded by $u_{1}, v_{i}, u_{2}, v_{i+1}$ $\left(v_{k+1}=v_{1}\right)$, add an edge joining $w_{i}$ to $u_{1}$ and to $u_{2},\left\lfloor\frac{g-2}{2}\right\rfloor$ parallel edges joining $w_{i}$ and $v_{i}$ and $\left\lceil\frac{g-2}{2}\right\rceil$ parallel edges joining $w_{i}$ and $v_{i+1}$, see Figure 1 for illustration.

This way we obtain the graph $G_{g}$ having $2 k$ vertices of degree $g$ and 2 vertices of degree $2 k$. It is easy to see that $g\left(G_{g}^{*}\right)=g$ and $\left|F\left(G_{g}\right)\right|=g \cdot k$. The vertices $w_{1}, \ldots w_{k}$ are face independent, thus $\alpha_{2}\left(G_{g}\right) \geq k$. Therefore, the inequality (2) holds.

For $g=1$ it suffices to add a vertex of degree one into each face of $G_{0}$. The resulting graph $G_{1}$ has $k$ faces, the girth of $G_{1}^{*}$ is 1 and the $k$ new vertices form a face independent set, therefore, the graph $G_{1}$ satisfies (2).

For $g=1,2,3$, and 4 , the graphs $G_{g}$ are simple. Graphs with $g>5$ cannot be simple, since every simple plane graph contains a vertex of degree at most 5 . For $g=5$ we can use a different construction to find examples of simple plane graphs such that the upper bound is attained.

Let us start with a $k$-sided antiprism $A_{k}$, subdivide each triangular face into three new triangles by adding a new vertex of degree three, delete the edges of the antiprism originally incident with two triangles and insert the other of the two diagonals in the new quadrangular faces. This way we get a 5-regular plane graph $H$ on $4 k$ vertices (see Figure 2 for illustration). Take the dual $H^{*}$ of $H$ and insert a new vertex of degree 5 into each face of $H^{*}$ and join it to all five vertices incident with the face. Let the resulting graph be $G_{5}^{\prime}$. It is easy to see that the girth of the dual of $G_{5}^{\prime}$ is 5 and that $\left|F\left(G_{5}^{\prime}\right)\right|=5 \cdot 4 k=20 k$. The $4 k$ newly inserted vertices form a face independent set in $G_{5}^{\prime}$, therefore $G_{5}^{\prime}$ satisfies (2).

Corollary 3.1 Let $G=(V, E, F)$ be a connected plane graph. If the dual $G^{*}$ has girth $g$ and there are disjoint cycles $C_{1}, \ldots, C_{t}$ of order $g$ that cover all the vertices of $G^{*}$ then $\operatorname{ls}(G)=t+2$.

\section{Proof}




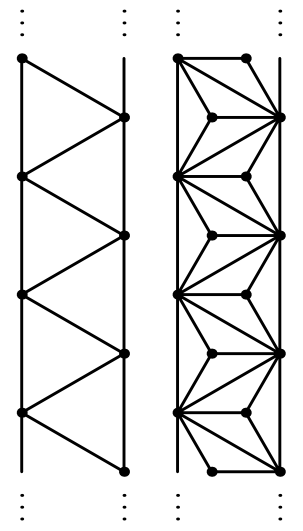

$A_{k}$

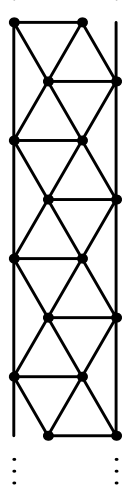

$H$

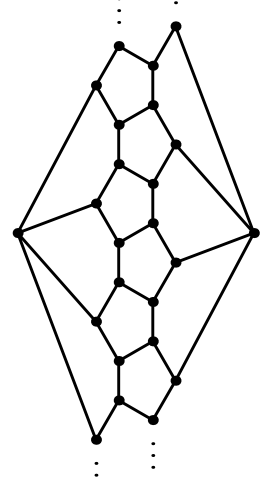

$H^{*}$

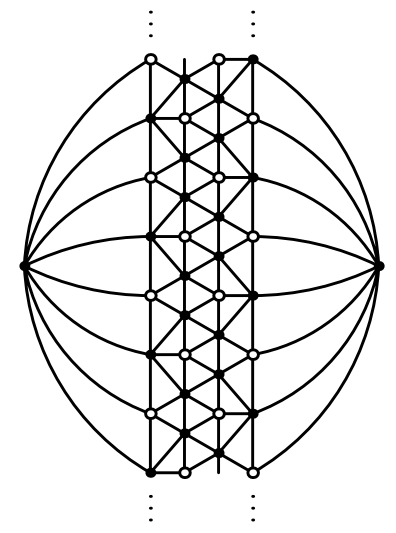

$G_{5}^{\prime}$

Figure 2: The construction of the graph $G_{5}^{\prime}$ from the graph of an antiprism.

The lower bound follows from Theorem 2.1. The upper bound is obtained from Theorem 3.1, because the number of vertices of $G^{*}$ is $t g$.

The edge connectivity of a plane graph $G$ plays an important role in the concept of the looseness of $G$. Observe, that each minimum edge-cut of size $g$ in $G$ corresponds to a cycle in $G^{*}$ and vice versa, therefore, the edge connectivity of a graph $G$ is equal to the girth of the dual graph $G^{*}$. Hence, we can obtain an upper bound for the looseness of plane graphs in terms of the edge connectivity of $G$.

Theorem 3.2 Let $G=(V, E, F)$ be a connected plane graph with the edge connectivity $\kappa^{\prime}$. Then

$$
l s(G) \leq \frac{1}{\kappa^{\prime}}|F(G)|+2 .
$$

Moreover, the bound is sharp.

Using Theorem 3.2 we can receive an upper bound for the looseness of 3connected cubic plane graphs.

Corollary 3.2 Let $G$ be an n-vertex 3-connected cubic plane graph. Then

$$
l s(G) \leq \frac{1}{6} n+\frac{8}{3} .
$$

Moreover, the bound is sharp.

\section{Proof}

We apply the Euler's polyhedral formula $|V(G)|+|F(G)|-|E(G)|=2$ and the fact that $2|E(G)|=3|V(G)|$. We obtain $|F(G)|=\frac{1}{2} n+2$. The edge connectivity of $G$ is three and after applying Theorem 3.2 we have

$$
l s(G) \leq \frac{1}{3}|F(G)|+2=\frac{1}{3}\left(\frac{1}{2} n+2\right)+2=\frac{1}{6} n+\frac{8}{3} .
$$


The sharpness follows from the following theorem.

Theorem 3.3 For any integer $t \geq 4$ there exists a 3-connected cubic plane graph $G=(V, E, F)$ on $n$ vertices such that

$$
t=l s(G)=\frac{1}{6} n+\frac{8}{3} .
$$

\section{Proof}

For $t=4$ the equation (3) yields $n=8$; it suffices to consider the graph of a cube. For $t=5$ we get $n=14$, one of the possible examples is depicted in Figure 3 .

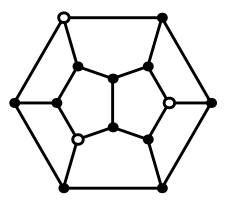

Figure 3: An example of graph on 14 vertices with $l s(G)=5$.

Let $t \geq 6$ and let $H$ be an arbitrary simple 3 -connected cubic plane graph on $2 t-8$ vertices (for example, $K_{4}$ or $(t-4)$-sided prism). The graph $H$ is cubic plane graph therefore $|F(H)|=t-2$.

We insert a star $K_{1,3}$ into each face of $H$ in the following way. Let $f$ be a face of $H$. We choose three edges on the boundary walk of the face $f$ and we put one vertex on each of them and put one "central" vertex to the interior of $f$. We join this "central" vertex with the other three added vertices. If we perform this operation on each face of $H$ we get a new graph $G$. The graph $G$ is 3 -connected cubic plane graph, furthermore, it has $n=2 t-8+4 \cdot(t-2)=6 t-16$ vertices.

Colour the "central" vertices of $G$ with distinct colours $1, \ldots, t-2$ and the remaining vertices with the same colour $t-1$. We obtain a nonloose $(t-1)$ colouring of $G$. Hence, $l s(G) \geq t$. On the other hand, from Corollary 3.2 we have

$$
l s(G) \leq \frac{1}{6}(6 t-16)+\frac{8}{3}=t,
$$

which proves the claim.

\section{Looseness and number of vertices}

Proposition 4.1 Let $G$ be a plane graph on $n$ vertices which contains a face incident with at least three vertices. Then $l s(G) \leq n$.

If $G$ is a simple plane graph then this trivial upper bound is tight if and only if $G$ is a triangle. If multiple edges or loops are allowed, suitable graphs could be constructed easily. 
Now we show that if $G$ is a simple connected plane graph on $n$ vertices, $n \geq 4$, then the number of colours in a nonloose colouring does not exceed $\frac{2 n+2}{3}-1$. This bound is the best possible for classes of 1-, 2-, and 3-connected plane graphs.

Observe, if a connected plane graph $G$ has 3 or 4 vertices then $l s(G)=3$.

Let $\mathcal{A}_{n}$ be a set of connected simple plane graphs on $n$ vertices, $n \geq 5$. Let

$$
\mathcal{B}_{n}=\left\{G \in \mathcal{A}_{n}: \quad \forall H \in \mathcal{A}_{n} \quad l s(G) \geq l s(H)\right\}
$$

be the set of the graphs on $n$ vertices with the maximal possible looseness; let its value be denoted by $t_{n}$. Let

$$
\mathcal{C}_{n}=\left\{G \in \mathcal{B}_{n}: \quad \forall H \in \mathcal{B}_{n} \quad|E(G)| \leq|E(H)|\right\}
$$

be the set of the graphs on $n$ vertices with the maximal possible looseness and minimal number of edges.

Lemma 4.1 Let $G$ be a graph from the set $\mathcal{C}_{n}$ and let $\varphi$ be a nonloose $\left(t_{n}-1\right)$ colouring of $G$. Then every heterochromatic edge of $G$ is a bridge.

\section{Proof}

Suppose there is a heterochromatic edge $e$ which is not a bridge. Let $H=G-e$ be a graph obtained from $G$ by deleting the edge $e$. Clearly, the graph $H$ is a connected plane graph on $n$ vertices and $l s(H)=l s(G)$. We have a contradiction with the fact that $G \in \mathcal{C}_{n}$.

Lemma 4.2 There is a graph $W \in \mathcal{C}_{n}$ and its nonloose $\left(t_{n}-1\right)$-colouring $\varphi$ such that all the vertices contained in at least one cycle have the same colour.

\section{Proof}

Let $G \in \mathcal{C}_{n}$ and let $\varphi$ be a nonloose $\left(t_{n}-1\right)$-colouring of $G$. Assume that $G$ contains at least two vertex disjoint cycles. We choose such cycles $C_{1}$ and $C_{2}$ that the distance between $C_{1}$ and $C_{2}$ is the smallest possible. From Lemma 4.1 follows that each vertex on $C_{1}$ has the same colour $i$ and each vertex on $C_{2}$ has the same colour $j$. Assume that $i \neq j$. The graph $G$ is connected, hence there is a path between $C_{1}$ and $C_{2}$. Observe that each vertex on the shortest path between $C_{1}$ and $C_{2}$ has a colour from the set $\{i, j\}$ (otherwise, there would be either a loose face or another pair of cycles in a smaller distance). Hence, every such path contains at least one heterochromatic edge $u v$. From Lemma 4.1 follows that $e$ is a bridge in $G$.

Now we define a new graph $H$ and a vertex colouring $\widetilde{\varphi}$ of $H$ in the following way. Let $G / u v$ be the graph obtained when contracting the edge $u v$ and let $z$ be a new vertex so obtained.

$$
\begin{aligned}
& V(H)=V(G / u v) \cup\{w\}, \quad E(H)=E(G / u v) \cup\{z w\} . \\
& \widetilde{\varphi}(x)=\left\{\begin{aligned}
\varphi(x) & \text { if } x \neq z, w \text { and } \varphi(x) \neq j, \\
i & \text { if } \varphi(x)=j, \\
i & \text { if } x=z, \\
j & \text { if } x=w .
\end{aligned}\right.
\end{aligned}
$$




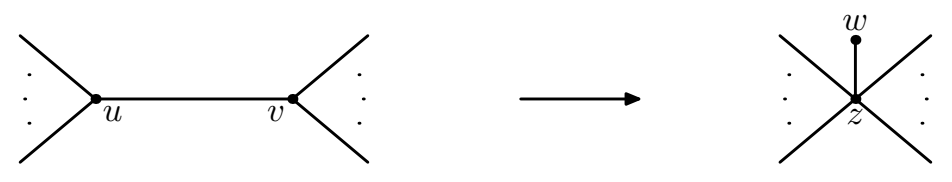

Figure 4: The new graph $H$ from the graph $G$

Clearly, $l s(H)=l s(G)=t_{n}$. If we use this method of recolouring of cycles we can find a graph $W \in \mathcal{C}_{n}$ such that vertices of all cycles have the same colour.

Lemma 4.3 There is a graph $G$ in the set $\mathcal{C}_{n}$ and a nonloose $\left(t_{n}-1\right)$-colouring $\varphi$ of $G$ such that each vertex on each cycle has the same colour 1 and for each colour from the set $\left\{2, \ldots, t_{n}-1\right\}$ there is a leaf $v_{i} \in V(G)$ such that $\varphi\left(v_{i}\right)=i$.

\section{Proof}

From Lemma 4.2 follows that there is a graph $W \in \mathcal{C}_{n}$ and a nonloose $\left(t_{n}-1\right)$ colouring $\varphi_{1}$ of $W$ such that each vertex on each cycle has the same colour, say 1. (Since $W \in \mathcal{C}_{n}$ and $n \geq 5$ the graph $W$ contains at least one cycle.)

Let $i$ be a colour from the set $\left\{2, \ldots, t_{n}-1\right\}$. Each vertex with colour $i, i \neq 1$, does not lie on any cycle in $W$. Suppose that there is no leaf with colour $i$. Every vertex coloured by $i$ is incident only with bridges, therefore, its neighbours have colours from the set $\{1, i\}$ (else there is a face which uses three different colours). Let $u v$ be an edge such that $\varphi_{1}(u)=i$ and $\varphi_{1}(v)=1$.

Now we define a new graph $H$ and a vertex colouring $\varphi_{2}$ of $H$ in the following way. Let $z$ be a vertex which we get when contracting the edge $u v$.

$$
\begin{gathered}
V(H)=V(G / u v) \cup\{w\}, \quad E(H)=E(G / u v) \cup\{z w\} . \\
\varphi_{2}(x)=\left\{\begin{aligned}
\varphi_{1}(x) & \text { if } x \neq z, w, \\
1 & \text { if } x=z, \\
i & \text { if } x=w .
\end{aligned}\right.
\end{gathered}
$$

Observe that $l s(H)=l s(G)=t_{n}$. Moreover, the vertex $w$ is a leaf and $\varphi_{2}(w)=i$. Using this method of recolouring of vertices we can find a graph $G$ with the desired properties.

Theorem 4.1 Let $G$ be a connected simple plane graph on $n$ vertices, $n \geq 4$. Then

$$
l s(G) \leq \frac{2 n+2}{3}
$$

Moreover, the bound is sharp.

\section{Proof}

The case when $n=4$ is trivial. Assume that $n \geq 5$. Let $H$ be a graph from $\mathcal{C}_{n}$ and $\varphi$ be a nonloose $\left(t_{n}-1\right)$-colouring of $H$ such that each vertex on each cycle 
has the same colour 1 and for each colour $i, i \in\left\{2, \ldots, t_{n}-1\right\}$ there is a leaf $w_{i}$ such that $\varphi\left(w_{i}\right)=i$. Since $H \in \mathcal{C}_{n}$ we have $l s(G) \leq l s(H)=t_{n}$.

In a graph $H$, there is a face $\alpha_{i}$ such that $w_{i} \in \alpha_{i}$ for every $i \in\left\{2, \ldots, t_{n}-1\right\}$. Since each face is incident with at least one vertex coloured with the colour 1 , the faces $\alpha_{i}$ are pairwise distinct. Therefore, $|F(H)| \geq t_{n}-2$.

Let $W$ be a graph obtained from $H$ by deleting all the leaves. Then

$$
|V(W)| \leq n-\left(t_{n}-2\right)
$$

The graph $W$ is a simple connected plane graph, therefore,

$$
\left.|F(W)| \leq 2|V(W)|-4 \leq 2\left(n-t_{n}+2\right)\right)-4=2 n-2 t_{n} .
$$

On the other hand, $|F(W)|=|F(H)| \geq t_{n}-2$, hence

$$
\begin{aligned}
2 n-2 t_{n} & \geq|F(W)| \geq t_{n}-2, \\
t_{n} & \leq \frac{2 n+2}{3} .
\end{aligned}
$$

The sharpness of this bound follows from the next theorem.

Theorem 4.2 For any integer $t \geq 1$ and any $k \in\{1,2,3\}$ there exists a simple $k$-connected plane graph $G=(V, E, F)$ on $n$ vertices, $n \geq t$, such that

$$
l s(G)=\frac{2 n+2}{3} .
$$

\section{Proof}

Let $k \in\{1,2,3\}$. Let $T$ be an arbitrary (3-connected) triangulation on $t$ vertices. We insert a new vertex into each face of $T$. We join each new vertex with $k$ vertices of a corresponding face. We obtain a $k$-connected plane graph $G_{k}$.

The graph $G_{k}$ has $t+(2 t-4)=3 t-4$ vertices. If we colour the vertices of $T$ with the same colour 1 and the other vertices with colours $2, \ldots, 2 t-3$ we obtain a nonloose $(2 t-3)$-colouring of $G_{k}$ for $k \in\{1,2,3\}$. Hence $l s\left(G_{k}\right) \geq 2 t-2$.

$$
l s\left(G_{k}\right) \geq 2 t-2=\frac{2}{3}(3 t-4)+\frac{2}{3}=\frac{2}{3}\left|V\left(G_{k}\right)\right|+\frac{2}{3}=\frac{2\left|V\left(G_{k}\right)\right|+2}{3} .
$$

The reverse inequality follows from Theorem 4.1.

\section{Looseness of triangulations}

Remind that the rainbowness of a plane graph $G$ is the minimum number of colours $r b(G)$ that any surjective vertex colouring of $G$ with $r b(G)$ colours enforces a face with vertices of mutually distinct colours. Clearly, every loose colouring of a triangulation $G$ is also a rainbow colouring, hence, $r b(G)=l s(G)$. 
The yet known upper bound for the rainbowness of plane triangulations has been $2 \alpha_{0}+1$, where $\alpha_{0}$ is the independence number. Now we improve it to $\frac{n+2}{2}$ if the minimum vertex degree is 4 and $\frac{2 n+6}{5}$ if the minimum vertex degree is 5 , where $n$ is the number of vertices. For triangulations with vertices of degree 3 the bound is $\frac{2 n+2}{3}$.

In the following let $\delta(G)$ denote the minimum vertex degree of $G$.

Theorem 5.1 Let $G$ be a simple plane triangulation. Then

$$
l s(G) \leq \frac{2|V(G)|-4}{\delta(G)}+2 .
$$

\section{Proof}

From the Euler's polyhedral formula $|V(G)|+|F(G)|-|E(G)|=2$ and the fact that $2|E(G)|=3|F(G)|$ we can derive $|F(G)|=2|V(G)|-4$. It is easy to show that if the minimum vertex degree of a simple triangulation is $g, g \in\{3,4,5\}$, then the girth of the dual graph is also $g$. The result follows from Theorem 3.1.

Corollary 5.1 Let $G$ be a simple n-vertex plane triangulation. Then

(i) $l s(G) \leq \frac{2 n+2}{3}$ if $\delta(G)=3$,

(ii) $l s(G) \leq \frac{n+2}{2}$ if $\delta(G)=4$,

(iii) $l s(G) \leq \frac{2 n+6}{5}$ if $\delta(G)=5$.

Moreover, the bounds are tight.

\section{Proof}

The claim follows immediately from Theorem 5.1. To see that these bounds are sharp we consider the graph $G_{3}$ from the proof of Theorem 4.2 if $\delta(G)=3$ and the graphs $G_{4}$ and $G_{5}^{\prime}$ from the proof of Theorem 3.1 if the minimum vertex degree is 4 or 5 , respectively.

Plane graphs are 4-colourable, hence there is a partition of vertices to 4 independent sets. From the pigeonhole principle follows that at least one of these sets have size at least $\left\lceil\frac{n}{4}\right\rceil$. Therefore, the independence number of each plane graph is at least $\left\lceil\frac{n}{4}\right\rceil$ (if a graph is 3-colourable then its independence number is at least $\left.\left\lceil\frac{n}{3}\right\rceil\right)$.

It is easy to see that $\frac{2 n+6}{5} \leq \frac{n+2}{2} \leq 2 \alpha_{0}+1$, hence the bounds in Corollary 5.1 improve the result of Negami.

Finally, we note that there are triangulations where the bound $\frac{2 n+2}{3}$ is smaller than $2 \alpha_{0}+1$ and vice versa, but it is well known that the problem to compute the independence number of plane graphs is NP-hard.

Acknowledgments: This work was supported by the Slovak Science and Technology Assistance Agency under the contract No APVV-0007-07. 


\section{References}

[1] K. Appel and W. Haken, Every planar map is four colorable, Contemp. Math. 98 (1989).

[2] J. A. Bondy and U. S. R. Murty, Graph theory, Springer, 2008.

[3] Z. Dvořák and D. Král, On planar mixed hypergraphs, Electron. J. Combin. 8(1) (2001) \#R35.

[4] Z. Dvořák, D. Král and R. Škrekovski, Non-rainbow colouring 3-, 4- and 5-connected plane graphs, J. Graph Theory (to appear).

[5] M. R. Garey, D. S. Johnson, Computers and Intractability, A Guide to the Theory of NP-Completeness, W. H. Freeman and Company, New York, 1979.

[6] S. Jendrol, Rainbowness of cubic polyhedral graphs, Discrete Math. 306 (2006) 3321-3326.

[7] V. Jungič, D. Král and R. Škrekovski, Colouring of plane graphs with no rainbow faces, Combinatorica 26(2) (2006) 169-182.

[8] D. Kobler and A. Kündgen, Gaps in the chromatic spectrum of faceconstrained plane graphs, Electron. J. Combin. 8(1) (2001) \#N3.

[9] A. Kündgen, E. Mendelsohn and V. Voloshin, Colouring planar mixed hypergraphs, Electron. J. Combin. 7 (2000) \#R60.

[10] S. Negami, Looseness ranges of triangulations on closed surfaces, Discrete Math. 303 (2005) 167-174.

[11] S. Negami and T. Midorikawa Loosely-tightness of triangulations of closed surfaces, Sci. Rep. Yokohama Nat. Univ., Sect. I. Math. Phys. Chem. 43 (1996) 25-41.

[12] J. G. Penaud, Une propriété de bicoloration des hypergraphes planaires, Cahiers Centre Études Rech. Opér 17 (1975) 345-349.

[13] R. Ramamurthi and D. B. West, Maximum face-constrained colouring of plane graphs, Electronic Notes in Discrete Math. 11 (2002) 542-549.

[14] R. Ramamurthi and D. B. West, Maximum face-constrained colouring of plane graphs, Discrete Math. 274 (2004) 233-240. 